\title{
EDITORIAL
}

DOI: $10.34057 /$ PPj.2020.39.01.001

Pelviperineology 2020;39(1):2

\section{Conservative management of pelvic pain and dysfunction}

It's a pleasure to introduce the first Pelviperineology issue for 2020. The focus of this special issue is on conservative management of chronic pelvic pain and dysfunction. Pelvic syndromes represent a constellation of disorders that are complex, span various specialties and require extensive knowledge and expertise for effective management. To advance the management of these conditions, the contributing authors share their knowledge, experience and practical solutions highlighting the role of complimentary therapies. As we begin the new decade it's important to reappraise the developments of recent years and note what they may foreshadow in terms of future trends. Several points deserve a mention.

There is a very evident paradigm shift in the classification, diagnosis and management of pelvic pain syndromes. The role of myofascial pain is more widely recognized. The most recent American College of Obstetricians and Gynecologists Practice Bulletin on Chronic Pelvic Pain (Number 218)* reflects this trend. Today, few if any, would subscribe to the diseased organ hypothesis as an explanation of chronic pelvic pain syndromes. In the past, interventions based on such presumptions were not only ineffective, but at times harmful. Current opinion considers the organ to be an innocent bystander, but the organs' immediate environment - the collagenous soft tissue, its malleability, elasticity and tensional balance, are in focus and receive far greater scrutiny. The association between dysfunctional muscle states and pelvic disorders has been well established and accepted, but what does it tell us in relation to pain and organ dysfunction? What are the actual mechanisms by which non-relaxing or overly relaxed muscles lead to dysfunction in organs and systems? Evidence points to the fascial system as the overlooked and mediating variable that regulates visceral function. With this shift in focus, first line interventions and therapies are being reconsidered and new approaches developed.

It is fortuitous that one of the new frontiers in the study of anatomy is the body-wide fascial system. In appraising pelvic disorders attention needs to be directed to ligaments, muscles, joints, fascia and viscera. Given that fascia is the primary communicator of mechanical information it is clearly implicated in the mechanisms of pain. The central location of the pelvis makes it an area of tensional convergence between the upper trunk and the lower limbs. The continuity of fascia which links the abdominal, pelvic and lumbar regions with the upper and lower extremities, paves the way for understanding why abdominal incisions and lumbar injuries are frequently associated with pelvic and bladder symptoms.
What is also interesting to note is that the fine tensional balance within the collagenous network impacts the highly reactive microscopic ganglia embedded in fascial architecture. These are the ganglia that control the peristalsis of each organ. Peristalsis is a local phenomenon. Consequently, injury, scars, adhesions, infections, recurrent inflammation, hormonal fluctuations, hydration, inactivity, muscle overactivation and emotional stress, all directly impact the dynamics of the fascial system and the function of organs. This role of the fascial mechanism is not yet adequately understood and deserves extensive study.

Conceptually, how does this impact the management of pelvic pain syndromes? If we consider that the pelvic region is subject to some of the most invasive medical procedures, ranging from caesarean sections, pelvic repair surgeries, synthetic mesh, tension tapes, grafts and bulking agents, laser treatments, and a range of -ectomies (hysterectomies, cystectomies, vestibulectomies and colectomies...), as well as the many laparoscopies investigating suspected endometriosis and organ disease, all of these directly impact the fine dynamics of the fascial system. When the malleability and elasticity of fascia are affected, it undoubtedly becomes implicated in visceral disorders and pain syndromes. With this in mind, the general appraisal of patients should include a functional neuromuscular assessment as well as an assessment of fascial dynamics.

A new mechanisms-oriented perspective on pain would be a sign of progress. Minds open to change pave the way to success and new beginnings. In this issue we begin with research on pain mapping, followed by a discussion on the mechanisms of central and peripheral sensitization, and new research on the role of biofeedback and magnetic stimulation in pelvic rehabilitation. These are complimented by two excellent case studies highlighting the application of the Stecco method of Fascial Manipulation. Each author generously shares valuable insight and information, contributing to existing knowledge. For every clinician, an evidenced based practice is power, making the management of pelvic syndromes more effective. I wish all of the recipients of Pelviperineology insightful reading.

Correspondence:

Marek Jantos PHD

Adelaide, Australia

marekjantos@gmail.com

*Chronic pelvic pain. ACOG Practice Bulletin No. 218. American College of Obstetricians and Gynecologists. Obstet Gynecol 2020;135:e98-109. 